\title{
Dynamic Service Reallocation in NFV-based Transport WDM Optical Networks
}

\author{
Albert Pagès \\ Optical Communications Group \\ Universitat Politècnica de Catalunya \\ Barcelona, Spain \\ albertpages@tsc.upc.edu \\ Salvatore Spadaro \\ Optical Communications Group \\ Universitat Politècnica de Catalunya \\ Barcelona, Spain \\ spadaro@tsc.upc.edu
}

\author{
Fernando Agraz \\ Optical Communications Group \\ Universitat Politècnica de Catalunya \\ Barcelona, Spain \\ agraz@tsc.upc.edu
}

\author{
Rafael Montero \\ Optical Communications Group \\ Universitat Politècnica de Catalunya \\ Barcelona, Spain \\ rafael.montero@tsc.upc.edu
}

\begin{abstract}
Future network services are expected to deliver additional functionalities besides connectivity. The Network Function Virtualization (NFV) paradigm provides the foundation for such concept. We propose a reallocation-based provisioning mechanism to enrich service provisioning architectures in NFV-based transport optical networks, achieving up to three orders of magnitude reductions in experienced blocking.
\end{abstract}

Keywords-Network Function Virtualization, slicing, optical transport networks.

\section{INTRODUCTION}

Emerging advanced services (e.g. massive Internet of Things (IoT), eHealth) impose requirements that challenge the capacity of infrastructure and service providers to efficiently deliver them towards customers. Such network services (NSs) are expected to not only deliver connectivity between distributed end-points but also richer functionalities (e.g. traffic shaping, pattern recognition) that are hard to accommodate with current rigid architectures.

The Network Function Virtualization (NFV) [1] paradigm is seen as the solution for efficient and flexible NS provisioning. Network functions are removed from core hardware and deployed in the form of software appliances, named Virtual Network Functions (VNFs), in compute resources (i.e. Data Centers (DCs)). This enables the customization of such functions to deliver specialized NSs.

$\mathrm{NFV}$ is seen as one of the main enablers of the global $5 \mathrm{G}$ vision [2]. A key use case for $5 \mathrm{G}$ network infrastructures relates to the delivery of specialized NSs towards vertical customers, which encompass network, computing and service functions resources as foundation to provide services to third parties. To support them, a Network as a Service (NaaS) model is envisioned. Thus, a common network can be leveraged by different verticals, partitioning the infrastructure in self-contained slices to support different NSs [3], [4], with NFV providing the capacity to give different flavors for slice customization.

Such slices will span different infrastructure segments and technological domains. Hence, efficient ways to deliver slices compliant with end-to-end (e2e) NS requirements in each one of them are needed. In such scenario, transport optical networks and their virtualization are entertained to be part of the whole NS delivery process, interconnecting far apart access domains or/and multiple network operators. Thus, architectures and techniques to provision NSs and slices in NFV-based transport networks are a must and currently being heavily investigated [5]. In this work, we propose and evaluate a load balancing-based allocation mechanism, enriched with reallocation techniques, to be leveraged in architectural proposals for NFV-based Wavelength Division Multiplexing (WDM) transport optical networks, with the aim to enhance NS provisioning.

\section{Network SERVice Allocation IN NFV-BASEd OpticAl NETWORKS}

The considered scenario consists in a fully transparent WDM circuit switched optical network with some DCs connected to optical nodes to provide computational capacity in the form of Computational Units (CUs) to support the deployment of VNF instances for NS provisioning between $\mathrm{e} 2 \mathrm{e}$ points. As a general approach, NSs are defined as a set of chained VNF instances that must be deployed onto DC resources, with optical connections providing the necessary e2e network connectivity.

The architecture to support the NS deployment is based on the ETSI NFV Management and Orchestration (MANO) architecture [6], which provides a framework to support the deployment of NSs composed of VNF instances and manage their lifecycle. Fig.1 depicts a schematic of the assumed MANO architecture and its main components, along with the physical infrastructure and examples of deployed NSs. From top to bottom the main components are: NFV Orchestrator (NFVO), VNF Manager (VNFM) and Virtual Infrastructure Manager (VIM), here separated into IT and network VIMs (I-VIM and N-VIM, respectively).

The main responsibility of the NFVO is the handling and offering of the NSs, along with the handling of the different VNFMs. In general, a VNFM is responsible for the instantiation and management of the VNF instances that constitute a NSs, also providing cataloguing capabilities to describe VNF characteristics and behaviors, which can be consumed by the NFVO for advertisement towards NS consumers. To provision the necessary resources for VNF creation and chaining, the VNFM leverages on the capabilities of the VIMs, which are the managers and controllers as well as virtualizers of the underlying physical infrastructure, allowing to configure the computational resources (I-VIM) and network connectivity (N-VIM), that is, the DCs and the optical circuits, respectively, for the NSs. 


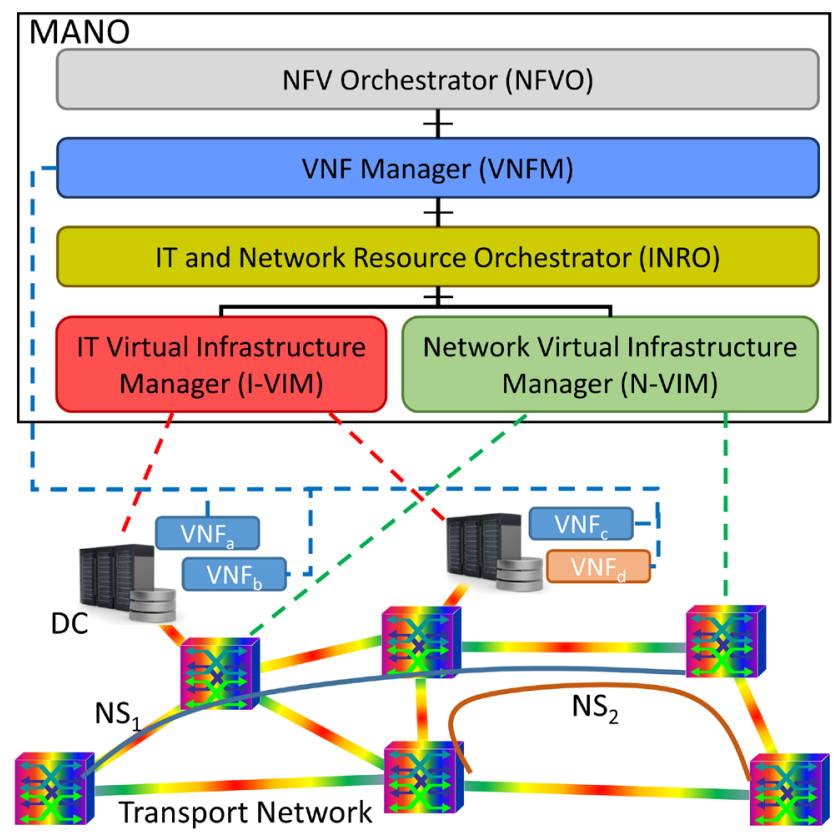

Fig. 1. NFV-based optical transport network architecture

Lastly, as a complement of the MANO architecture, the IT and Network Resource Orchestration (INRO) component is defined. The mission of such component is to, in the presence of heterogeneous resources, coordinate the different VIMs to determine the best resource layout as a way to achieve a holistic NS deployment. The inclusion of this component has been proposed in research works [5] as well as in software implementations of the MANO architecture (e.g. Open Source MANO (OSM).) It is in this component where we propose a NS provisioning mechanism to expand its functionalities towards enhanced service acceptance. The proposed mechanism is divided onto two sub-mechanisms: one for new NS allocation and another for reallocation of existing NSs when a new NS cannot be provisioned given current resource availability.

For the new NS allocation, a load balancing provisioning is followed. Firstly, all potential paths between source and destination are calculated, restricted to ones that include DCs connected to optical nodes. Then, the aggregated $\mathrm{CU}$ capacity of the NS is divided between the aggregated CU capacity of DCs in the route. For every source/destination-toDC and DC-to-DC segment in the route, the average ratio of requested wavelengths by the NS between continuous wavelengths in the first $\mathrm{K}$ ' shortest path between the nodes is calculated and added to the previous metric. Paths are then ordered in descending order given the metric. Next, the first $\mathrm{K}$ paths are explored for NS allocation. VNF instances are allocated iteratively onto the first DC along the candidate route with enough CUs to host them, potentially resulting in VNFs hosted onto the same or different DCs. Once placed, required wavelengths are assigned in a first fit criterion. Note that, while a fully transparent transport network is considered, hence wavelength continuity must be respected for source/destination-to-DC and DC-to-DC route segments, optoelectronic conversion capabilities are present at DC nodes to allow for the injection/extraction of traffic towards/from the hosted VNF instances, thus different segments may employ different sets of wavelengths.

As for the NS reallocation mechanism, it is triggered when a NS cannot be accommodated following the previous strategy. In such situation, the proposed mechanism leverages on the capacity of existing VNFMs and VIMs (e.g. OSM, OpenStack) to migrate/re-configure resources to reallocate active NSs and make room for new ones. The coordination of the necessary operations is achieved through the INRO module. The procedure for NS reallocation is as follows: active NSs that share some resources with candidate paths are determined, and paths are ordered ascendingly according to this. For each of them, the active NSs are ordered descendingly following a combination of IT and network occupancy. Starting from the largest, an alternative resource allocation is determined following the aforementioned load balancing mechanism, forbidding the previous allocation. If found, resources are released and the new NS may be served. Note that, if despite the calculated NS reallocations, the new NS cannot be served in the candidate path, the INRO does not trigger any reallocation, thus previous resource allocation for active NSs is respected.

\section{Mechanism Evaluation AND Discussion}

In this section, we will evaluate the performance of the proposed mechanism. To this end, we focus on a dynamic scenario where NSs arrive following a Poisson process. Fig. 2 depicts the employed network topology, with dark nodes denoting where DCs are connected. We assume 80 wavelengths per fiber link, with each DC equipped with $10^{5}$ CUs and arbitrarily large network capacity towards the transport network. Then, NSs are uniformly distributed among all pairs of source/destination nodes. Each one of them requests for a sequence of 1-4 VNF instances, with each instance requiring between 100-1000 CUs in steps of 100 , and an e2e network capacity of 1-3 wavelengths.

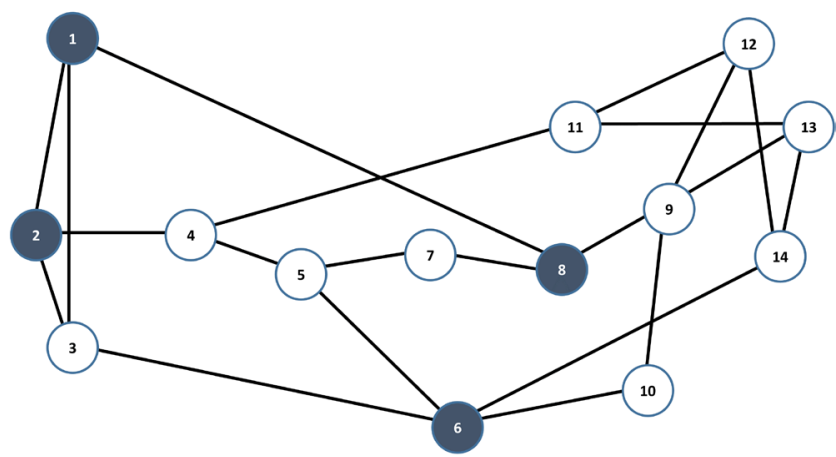

Fig. 2. Assumed transport network topology and DC location.

First, we evaluate the blocking probability (BP) experienced by incoming NSs as a function of their normalized average holding time (HT) (Fig. 3), assuming an average inter-arrival time between service requests of one time unit. As benchmarks, we employ a variation of the mechanism where reallocations are not allowed, as well as a greedy allocation mechanism in which NSs are allocated onto the first shortest path (in hops) that includes DC nodes and has enough resources, both network and computing, to allocate the VNF instances as well as the desired e2e capacity. In all results, $\mathrm{K}$ is set to 3 candidate paths and $10^{5}$ random NS requests are considered.

It can be seen how the proposed mechanism substantially reduces the BP, especially with reallocation techniques. This translates to an increasing capacity to sustain higher loads for the same BP. For example, for a $\mathrm{BP}=10^{-3}$, around increases of 1.97 and 2.25 times in sustained load are attained without 
and with reallocation, respectively. Conversely, focusing on the same load, reductions of up to two and three orders of magnitude in BP are achieved without and with reallocation, respectively, for lower loads while reductions of about 40 $60 \%$ are achieved for higher loads. Moreover, differences up to two orders of magnitude when comparing the mechanisms without and with reallocation are observed. All of this highlights that reallocation techniques can be beneficial in NFV-based optical networks.

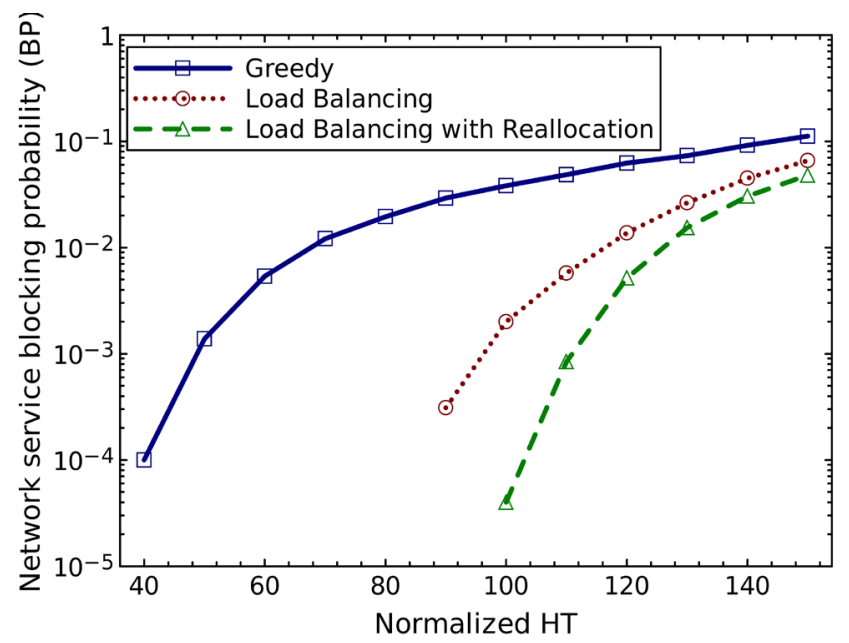

Fig. 3. Blocking probability of network services as a function of the normalized holding time.

However, reallocation techniques come to the price of potentially having to reallocate a significant number of active NSs, affecting both established optical connections and VNF instances. To analyze this, we extracted the average number of service, e2e connection and VNF instance reallocations per accepted NS in the network. In this regard, note that such metrics also account for the events in which a new NS can be allocated given actual free resources, thus not requiring to disturb already allocated NSs, as well as the cases in which a new NS requires the reallocation of multiple active NSs to make room for it. Taking averages from all the cases provides an estimation about the number of reallocations to be performed for new service requests.

Let us also comment about the relationship between the three metrics. We consider that a NS is reallocated if either the original location of its VNF instances is changed or the characteristics of the e2e optical connections assigned to it are altered. The VNF reallocation metric accounts for the individual number of VNFs that are required to change location, either if they belong to the same NS instance or multiple ones. On the other hand, connection reallocations account for any change on the individual lightpaths allocated to active NSs. These changes may be due to having to select alternative routes for source/destination-to-DC and DC-toDC spans, changing the wavelength for the spans or a combination of both. Additionally, there may be situations in which, although the e2e route (sequence of fiber links) is maintained, as well as the assigned wavelength channels, VNF locations have changed among DCs in the route, thus points of traffic injection/extraction have also changed, requiring to reconfigure the involved optical nodes. Any of these events is considered as a connection reallocation and accounted in the metric. Hence, note that a service reallocation always entails the reallocation of its optical connections, while VNF reallocations may happen or not depending on the needs of freeing CUs availability in specific DCs. Given this considerations, the obtained results are depicted in Fig. 4.

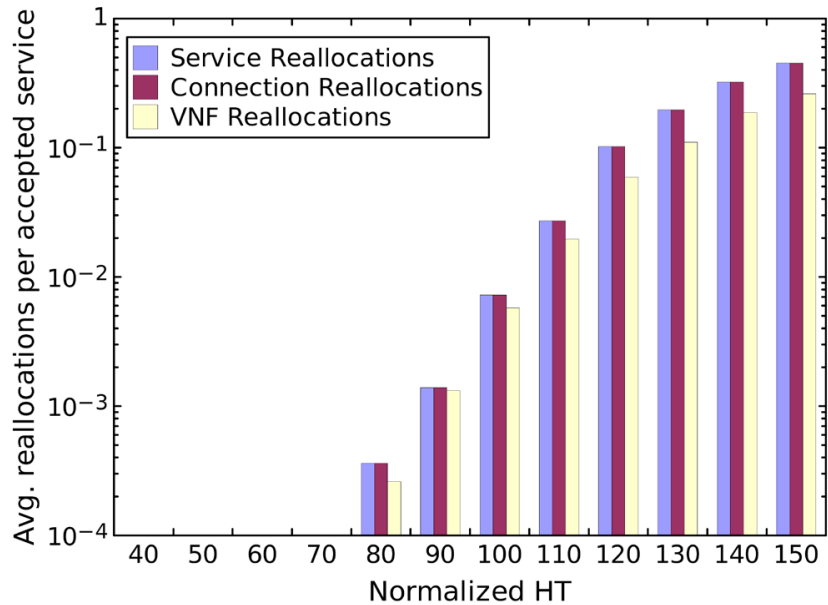

Fig. 4. Average service, connection and VNF instance reallocations per accepted service as a function of the normalized holding time.

We can see that the required number of reallocations is very small, ranging from around $3.6 \cdot 10-4$ to 0.5 reallocations per accepted NS, meaning that the number of affected active NSs is few and the majority of new NSs do not entail any reallocation. This is because the proposed mechanism distributes the NSs across the physical infrastructure, thus the number of active NSs sharing resources is minimized. Moreover, the mechanism takes into account such number when deciding necessary reallocations. As a result, the percentage of new NSs that require to perform reallocation operations is very small ( $4 \%$ in the worst case).

\section{CONCLUSIONS}

We proposed a load balancing-based mechanism with reallocation techniques to be leveraged in NFV-based optical transport networks as part of standard architecture designs for NS provisioning. Results reveal that significant improvements in blocking levels can be achieved with minimal reallocation costs.

\section{ACKNOWLEDGMENT}

This work has been supported by the H2020 5GPPP SLICENET project (H2020-ICT-2016-2/761913) and the Spanish Government through project ALLIANCE (TEC2017-90034-C2-2-R) with FEDER contribution.

\section{REFERENCES}

[1] ETSI, "Network Function Virtualization - White Paper no. 3," ETSI White Paper, October 2014

[2] ETSI, "Network Function Virtualization - White Paper on NFV priorities for 5G," ETSI White Paper, February 2017.

[3] SliceNet 5GPPP project, https://slicenet.eu

[4] IETF, "Common Operations and Management on Network Slices (COMS) Architecture,” IETF Internet Draft, March 2018.

[5] R. Vilalta, A. Mayoral, R. Muñoz, R. Casellas and R. Martínez, "Multitenant Transport Networks With SDN/NFV," IEEE Journal of Lightwave Technology, vol. 2, no. 6, pp. 1509-1515, March 2016.

[6] ETSI, "NFV Management and Orchestration," ETSI Group Specification, December 2014. 